investigate and visualise characteristic marker prevalence and coprevalence patterns.

Results Based on the individual marker pattern, patients can often be stratified belonging to different study subgroups. For example, for SLE we show that different reactivity groups exist including patients with different disease activity scores and organ damage patterns.

Conclusions We conclude that the approach of a comprehensive prevalence and signature analysis and a vivid data visualisation is useful for any multiplex omics assay.

\section{PS1:21 URINARY MARKERS OF INFLAMMATION IN LUPUS NEPHRITIS PATIENTS}

J Kosalka, B Jakiela, J Musial. Jagiellonian University Medical College, Department of Medicine, Krakow, Poland

\subsection{6/lupus-2018-abstract.69}

Objectives Renal involvement is the most important manifestation of systemic lupus erythematosus, but assessing of inflammatory response in kidneys with non-invasive methods is still challenging. In this study we aimed to define markers of active lupus nephritis (LN) using urine immune profiling.

Methods Levels of cytokines (18-plex array) and mRNA expression (40 immune and glomerular injury genes) were measured in urine samples of $\mathrm{LN}$ patients with active disease $(\mathrm{n}=17)$, during remission $(\mathrm{n}=16)$, and in healthy subjects $(\mathrm{n}=19)$.

Results Urine levels of CCL2, CCL5, CXCL10 and IL-6 were elevated in active LN as compared to remission (best discrimination for CCL2), and correlated with LN activity. In the active disease, urinary cell transcriptome showed strong upregulation of proinflammatory cytokines (e.g. TNF, CCL2, CCL5, CXCL10), Th1 related genes (e.g. CD3G, CD4, TBX21, IFNG), and markers of glomerular damage (NPHS2 [podocin]). Active pattern of gene expression was also observed in 5 patients in remission, who had moderately increased urinary leukocyte count, two patients from this group (40\%) developed renal exacerbation during following 3 months. Markers of Th17 immune axis (e.g. IL-17A) were not significantly increased in active LN.

Conclusions Active LN patients (also patients at risk of exacerbation) were characterised by marked increase of proinflammatory mediators in the urine. We identified CCL2 chemokine as the most promising marker for monitoring of disease flare.

\section{PS1:22 USE OF INTERFERON ALPHA AND INTERLEUKIN-10 AS CLINICAL ACTIVITY BIOMARKERS IN SYSTEMIC LUPUS ERYTHEMATOUS PATIENTS}

${ }^{1} \mathrm{E}$ Grau, ${ }^{2} \mathrm{M}$ Fernandez Matilla, ${ }^{1} \mathrm{CM}$ Feced Olmos, ${ }^{1} \mathrm{E}$ Labrador Sanchez, ${ }^{1} \mathrm{FM}$ Ortiz Sanjuan, ${ }^{2} \mathrm{~N}$ Fernandez-Llanio Cornella, ${ }^{1} \mid \mathrm{C}$ Chalmeta Verdejo, ${ }^{1} \mathrm{~K}$ Arevalo Ruales, ${ }^{1} \mathrm{R}$ Negueroles Albuixech, ${ }^{1} \mathrm{~J}$ Ivorra Cortes, ${ }^{1} \mathrm{JJ}$ Fragio Gil, ${ }^{1} \mathrm{I}$ Martinez Cordellat, ${ }^{1} \mathrm{R}$ Gonzalez Mazario, 'L Gonzalez Puig, ${ }^{1}$ C Alcañiz Escandell, ${ }^{1} \mathrm{C}$ Najera Herranz, 'I Canovas Olmos, ${ }^{1} E$ Vicens Bernabeu, ' JE Oller Rodriguez, ${ }^{1} \mathrm{M}$ de la Rubia Navarro, ${ }^{2} \mathrm{JA}$ Castellano Cuesta, ${ }^{3} \mathrm{~V}$ Fornes Ferrer, ${ }^{3} \mathrm{D}$ Hervas Marin, ${ }^{1} \mathrm{JA}$ Roman Ivorra. ${ }^{1}$ Rheumatology Department. HUP La $\mathrm{Fe}$, Valencia, Spain; ${ }^{2}$ Rheumatology Section. Hospital Arnau de Vilanova, Valencia, Spain; ${ }^{3}$ Biostatistics Unit. IIS La Fe, Valencia, Spain

\subsection{6/lupus-2018-abstract.70}

Purpose To analyse the association among INF1A, IL10 and BLyS levels and clinical activity in SLE.
Methods A cross-sectional, observational study of 142 patients diagnosed of SLE according to SLICC 2012 criteria and 34 healthy controls was performed. In patients a complete bloodtest was made, and clinical data by personal interview was collected. We analysed the serum concentration of IL10, BLyS and INF1A by colorimetric methods. SLE patients were dichotomized as high and low levels for each cytokine based on the cytokine level above $2 \mathrm{SD}$ of the mean in healthy controls. Biostatistical analysis with R (3.3.2.) was performed.

Results In our SLE patients we observed higher values of IL10, BLyS and INF1A than healthy controls $(p<0.001$, $\mathrm{p}=0.005$ and $\mathrm{p}=0.043$ respectively), showing an average values in patients of $13.39 \pm 27.73 \mathrm{pg} / \mathrm{mL}$ INF1A, $9.99 \pm 15.84 \mathrm{pg} / \mathrm{mL}$ IL10 and $1811.31 \pm 1757.81 \mathrm{pg} / \mathrm{mL}$ BLyS. The mean clinical activity measured by SLEDAI was $5.91 \pm 5.06$.

Statistical analysis indicate that INF1A levels are correlated to IL10 levels $(p=0.001)$ and BLyS levels $(p=0,034)$. Due to this finding, we categorised SLE patients by low or high level of the three cytokines: 44 INF1A(-)IL10(-)BLyS(-); 61 INF1A (+)IL10(-)BLyS(-); 5 INF1A(+)IL10(-)BLyS(+); 18 INF1A(+) $\operatorname{IL} 10(+) \mathrm{BLyS}(-)$ and $14 \operatorname{INF} 1 \mathrm{~A}(+) \operatorname{IL} 10(+) \mathrm{BLyS}(+)$. There is a high association of increased IL10-INF1A levels and the increased of clinical activity measured by SLEDAI score $(\mathrm{p}<0.0001)$, and to a lesser extent with increased IL10INF1A-BLyS levels. Patients with high IL10-INF1A and IL10INF1A-BLyS showed a significant rise in C3-C4 consumption $(p<0.001$ and $p=0.001$ respectively) and high anti-dsDNA $(p=0.001$ and $p=0.002$ respectively). Patients with increased INF1A-BLyS exhibited high anti-dsDNA $(p=0.004)$ and ENA positivity $(p<0.001)$. In addition, patients with increased levels of IL10-INF1A-BLyS showed ANAs $(\mathrm{p}<0.001)$ and antiphospholipid autoantibody positivity $(\mathrm{p}=0.004)$.

Conclusions The $69 \%$ of our SLE patients displayed almost one cytokine increased, being the INF1A the cytokine that mainly is increased. However, increased IL10 levels, irrespective of whether there is also increased levels of BLyS and/or INF1A, is the cytokine which best fits to clinical activity in SLE.

\section{Poster session 2: Autoantibodies, biomarkers and imaging (2), Environmental, epigenetics and genomics}

\section{PS2:23 ANTI-C1Q ANTIBODIES IN TURKISH SYSTEMIC LUPUS ERYTHEMATOSUS PATIENTS}

S Tekeoglu, D Temiz Karadag, O Ozdemir Isik, A Yazici, A Cefle. Kocaeli University, Internal Medicine, Rheumatology Department, Kocaeli, Turkey

\subsection{6/lupus-2018-abstract.71}

Purpose Anti-C1q has been shown to be associated with systemic lupus erythematosus (SLE) and disease activity of lupus nephritis in previous studies. We studied anti-C1q specificity for SLE versus rheumatic disease controls and healthy controls and the association with SLE manifestations in a single centre cross-sectional study.

Methods Demographics, disease information and blood samples were obtained during routine follow-up visits of patients attending Kocaeli University rheumatology outpatient clinic. There were 150 SLE patients (92\% female, mean: 46 years). Control group had 85 rheumatoid arthritis patients, 16 